\title{
Métodos de determinación de la cinética de hidratación mediante la retracción química y parámetros que lo influencian
}

\author{
Methods for determining hydration kinetics by chemical \\ shrinkage and parameters influencing it
}

\section{Delver Plinio Estrada Caceres Vanderley Moacyr John Maria Alba Cincotto}

Delver Plinio Estrada Caceres Universidade de São Paulo São Paulo - SP - Brasil

Vanderley Moacyr John Universidade de São Paulo São Paulo - SP - Brasil

Maria Alba Cincotto Universidade de São Paulo São Paulo - SP - Brasil

Recebido em 15/01/16 Aceito em 06/02/17

\section{Resumen}

$\mathbf{M}$

edir la retracción química proporciona información sobre el desarrollo de la cinética de hidratación de materiales cementicios; además está relacionada con el grado y calor de hidratación. Para determinar la retracción química, se cuantifica la variación de volumen debido a las reacciones de hidratación en una muestra de pasta de cemento en condiciones de saturación. En general existen tres formas para medir esta retracción: dilatometría, gravimetría y picnometría. Estos procedimientos experimentales requieren equipos sencillos y de bajo costo. Existen parámetros que pueden influenciar en los resultados, tales como: espesor de la muestra, relación agua/cemento, área superficial de la muestra, cantidad de agua sobre la muestra y composición del cemento. En este trabajo se detallan los métodos para determinar la retracción química en pastas de cemento Portland, así como los parámetros que influencian en los resultados.

Palabras clave: Cemento Portland. Retracción. Retracción química. Métodos de medición.
Abstract
Measuring chemical shrinkage provides information about the development of hydration kinetics of cementitious materials. It is also related to the degree and heat of hydration. To determine chemical shrinkage, volume change caused by the hydration reactions in a sample of cement paste under saturation conditions is quantified. There are three methods to measure this shrinkage: dilatometry, gravimetry and picnometry. These experimental procedures require simple and inexpensive equipment. There are parameters that can influence the results, such as sample thickness, water/cement ratio, surface area of the sample, amount of water on the sample and cement composition. In this research, the methods for measuring chemical shrinkage in Portland cement pastes are detailed, as well as the parameters that influence the results.
Keywords: Portland cement. Shrinkage. Chemical shrinkage. Measurement methods. 


\section{Introducción}

La retracción del concreto se presenta desde el contacto del cemento con el agua y se prolonga del estado fluido al endurecido. En los primeros momentos ocurre el consumo del agua de mezclado, en parte por las reacciones de hidratación responsables por la retracción química, la liberación de calor y la expansión térmica que, con su posterior enfriamiento, causa la retracción térmica. En un sistema cerrado ( $\sin$ intercambio de humedad con su entorno) y con baja relación agua/cemento, ocurre la retracción por auto-secado o retracción autógena, como consecuencia a las reacciones químicas. En un sistema de gran área superficial expuesta al aire no saturado predomina la evaporación del agua no combinada, responsable por la retracción por secado.

Medir la retracción química de materiales cementicios tiene propósitos múltiples, como: determinar el potencial de fisuración y la cantidad de vacíos internos del concreto (LURA et al., 2009); y cuantificar el grado y la cinética de hidratación (TAZAWA; MIYAZAWA; KASAI, 1995; MOUNANGA et al., 2006; ZHANG et al., 2010). Además, así como la retracción química está correlacionada con las reacciones de hidratación, también está relacionada con el calor liberado y puede ser utilizada para sustituir calorímetros isotérmicos o semi-adiabáticos. Medir la retracción química en comparación a los métodos de calorimetría es una tarea de simple ejecución, requiere equipo de bajo costo y permite mediciones a largo plazo, inclusive años. Los resultados de la cinética de hidratación se obtienen a partir de la variación de volumen (retracción química) y el flujo de calor (calorímetro) similares. Sin embargo, con este potencial, este método ha recibido poca atención de la comunidad técnica.

Por lo expuesto, este trabajo tiene como objetivo presentar los métodos que existen para medir la retracción química y las dificultades presentadas cuando se realizan las determinaciones experimentales.

\section{Retracción química}

La retracción química está asociada con las reacciones de hidratación del cemento debido a que el volumen de los productos hidratados es menor que el volumen inicial de los reactantes (agua, ligantes) (GEIKER; KNUDSEN, 1982; TAZAWA, 1999; JENSEN; HANSEN, 2001a). Su valor es expresado por una variación del volumen absoluto. La retracción química es relativamente menor en comparación a la retracción térmica y a la de auto secado (JENSEN; HANSEN, 2001a).
Esta retracción comenzó a ser estudiada por Le Chatelier, quien, alrededor de 1900, fue el primero en observar que la hidratación del cemento produce una reducción en el volumen absoluto de las pastas de cemento. En 1948 Powers usó la retracción química para cuantificar el grado de reacción química ocurrida, es decir, la usó como un indicador del progreso de hidratación. Por otra parte, Parrot et al. (1990) mencionan que la retracción química es una consecuencia inducida por la hidratación del cemento Portland, por lo tanto existe una relación directa entre ambas.

Cada fase anhidra del cemento tiene un coeficiente de retracción química característico, asociado a la cantidad de agua de reacción y a la organización cristalina original e hidratada. Estudios como los de Tazawa (1999), Bentz (1997), Bentz, Lura y Roberts (2005), Pang et al. (2013) y Yodsudjai y Wang (2013) demuestran que es posible predecir la cantidad de retracción química de forma analítica usando la Ecuación 1; que depende del coeficiente de retracción, la cantidad y el grado de hidratación, de cada fase anhidra del cemento Portland. Cada fase constituyente del cemento Portland tiene reacción química, cinética de hidratación y retracción química propia (Ecuación 1).

$R Q=\left[0.0704 \cdot \propto_{C_{3} S}(t) \cdot \% C_{3} S\right]+\left[0.0724 \cdot \propto_{C_{2} S}(t) \cdot\right.$

$\left.\% C_{2} S\right]+\left[0.171 \cdot \propto_{C_{3} A}(t) \cdot \% C_{3} A\right]+\lfloor 0.117$.

$\left.\propto_{C_{4} A F}(t) \cdot \% C_{4} A F\right]$

Donde: $0.0704,0.0724,0.171$ y 0.117 son los coeficientes de retracción química del $\mathrm{C}_{3} \mathrm{~S}, \mathrm{C}_{2} \mathrm{~S}$, $\mathrm{C}_{3} \mathrm{~A}, \mathrm{C}_{4} \mathrm{AF}$, respectivamente; $\alpha \mathrm{C}_{3} \mathrm{~S}(\mathrm{t}), \alpha \mathrm{C} 2 \mathrm{~S}(\mathrm{t})$, $\alpha \mathrm{C}_{3} \mathrm{~A}(\mathrm{t})$ y $\alpha \mathrm{C}_{4} \mathrm{AF}(\mathrm{t})$ son los grados de reacción del $\mathrm{C}_{3} \mathrm{~S}, \mathrm{C}_{2} \mathrm{~S}, \mathrm{C}_{3} \mathrm{~A}$ y $\mathrm{C}_{4} \mathrm{AF}$ para un edad de hidratación (t); y $\% \mathrm{C}_{3} \mathrm{~S}, \% \mathrm{C}_{2} \mathrm{~S}, \% \mathrm{C}_{3} \mathrm{~A}$ y $\% \mathrm{C}_{4} \mathrm{AF}$ representan la fracción de la masa inicial de las fases del cemento Portland. El grado de hidratación de cada fase a lo largo del tiempo puede ser encontrado en la publicación de Lea's (HEWLETT, 1998). Es también posible producir correlaciones empíricas entre la retracción química y el agua combinada para cementos de determinada composición.

La magnitud típica de la retracción química para un cemento Portland común y con una hidratación completa varía entre 0.06 y $0.07 \mathrm{~mL} / \mathrm{g}$ de cemento (LURA; JENSEN; VAN BREUGEL, 2003). Esta disminución de volumen se debe a que el agua tiene volumen específico menor cuando está adherida a un producto hidratado en comparación a cuando está libre en un medio líquido (COSTOYA, 2008). También se debe considerar que para hidratar $1 \mathrm{~g}$ de cemento se requieren $0.23 \mathrm{~g}$ de agua (JENSEN; HANSEN, 2001b; NEVILLE; BROOKS, 2007). En la Tabla 1 se muestran los resultados de retracción 
química obtenidos en diversos estudios con diferencias en la edad de hidratación, composición de cemento, relación agua cemento $(\mathrm{a} / \mathrm{c}) \mathrm{y}$ temperatura.

Tabla 1 - Retracción química para diferentes edades de hidratación y otros parámetros

\begin{tabular}{|c|c|c|c|c|c|c|}
\hline Tipo de Cemento & $\begin{array}{l}\text { Composición del } \\
\text { cemento }\end{array}$ & $a / c$ & $\begin{array}{c}\text { Temp. } \\
\left({ }^{\circ} \mathrm{C}\right)\end{array}$ & $\begin{array}{c}\text { Edad de } \\
\text { hidra- } \\
\text { tación } \\
\end{array}$ & $\begin{array}{c}\text { Retracción } \\
\text { Química } \\
\text { (mL/g) }\end{array}$ & Referencia \\
\hline $\begin{array}{l}\text { Cemento Portland } \\
\text { común }\end{array}$ & $\begin{array}{c}57.15 \% \mathrm{C}_{3} \mathrm{~S}, \\
7.77 \% \mathrm{C}_{2} \mathrm{~S}, \\
3.81 \% \mathrm{C}_{3} \mathrm{~A}, \\
5.95 \% \mathrm{C}_{4} \mathrm{AF}, \\
5.22 \% \text { gipso }\end{array}$ & 0.4 & 30 & $\begin{array}{c}24 \\
\text { horas }\end{array}$ & 0.030 & $\begin{array}{l}\text { Mounanga et } \\
\text { al. (2004) }\end{array}$ \\
\hline $\begin{array}{l}\text { Cemento Portland } \\
\text { Tipo I C } 150 \\
\text { (AMERICAN..., 2007) }\end{array}$ & $\begin{array}{l}60 \% \mathrm{C}_{3} \mathrm{~S} \\
12 \% \mathrm{C}_{2} \mathrm{~S} \\
12 \% \mathrm{C}_{3} \mathrm{~A} \\
7 \% \mathrm{C}_{4} \mathrm{AF}\end{array}$ & 0.3 & 23 & $\begin{array}{c}48 \\
\text { horas }\end{array}$ & 0.040 & $\begin{array}{l}\text { Sant, Lura y } \\
\text { Weiss } \\
(2006)\end{array}$ \\
\hline $\begin{array}{l}\text { Cemento Portland } \\
\text { común }\end{array}$ & $\begin{array}{l}62.89 \% \mathrm{C}_{3} \mathrm{~S} \\
9,12 \% \mathrm{C}_{2} \mathrm{~S} \\
6.79 \% \mathrm{C}_{3} \mathrm{~A} \\
9.54 \% \mathrm{C}_{4} \mathrm{AF}\end{array}$ & 0.4 & 20 & $\begin{array}{c}24 \\
\text { horas }\end{array}$ & 0.029 & $\begin{array}{l}\text { Bouasker et } \\
\text { al. }(2008)\end{array}$ \\
\hline $\begin{array}{l}\text { Cemento de pozo de } \\
\text { petróleo }\end{array}$ & $\begin{array}{c}63.94 \% \mathrm{C}_{3} \mathrm{~S}, \\
15.84 \% \mathrm{C}_{2} \mathrm{~S}, \\
0.57 \% \mathrm{C}_{3} \mathrm{~A}, \\
11.3 \% \mathrm{C} 4 \mathrm{AF}, \\
1.8 \% \text { gipso }\end{array}$ & 0.35 & 25 & $\begin{array}{c}72 \\
\text { horas }\end{array}$ & 0.030 & $\begin{array}{l}\text { Zhang et al. } \\
(2010)\end{array}$ \\
\hline $\begin{array}{l}\text { Cemento Portland } \\
\text { común }\end{array}$ & $\begin{array}{c}56.1 \% \mathrm{C}_{3} \mathrm{~S} \\
15.5 \% \mathrm{C}_{2} \mathrm{~S} \\
4.8 \% \mathrm{C}_{3} \mathrm{~A} \\
11.5 \% \mathrm{C} 4 \mathrm{AF}, 4 \% \\
\text { gipso }\end{array}$ & 0.5 & 20 & $\begin{array}{c}72 \\
\text { horas }\end{array}$ & 0.030 & $\begin{array}{l}\text { Lura, } \\
\text { Winnefeld y } \\
\text { Klemm } \\
(2010)\end{array}$ \\
\hline $\begin{array}{l}\text { Cemento Portland } \\
\text { común }\end{array}$ & $\begin{array}{l}62.01 \% \mathrm{C}_{3} \mathrm{~S} \\
11.13 \% \mathrm{C}_{2} \mathrm{~S} \\
8.11 \% \mathrm{C}_{3} \mathrm{~A} \\
8,45 \% \mathrm{C}_{4} \mathrm{AF} \\
6,56 \% \text { gipso }\end{array}$ & 0.4 & 20 & $\begin{array}{c}72 \\
\text { horas }\end{array}$ & 0,030 & $\begin{array}{l}\text { Mounanga et } \\
\text { al. }(2011)\end{array}$ \\
\hline $\begin{array}{l}\text { Cemento Portland } \\
\text { Tipo I C } 150 \\
\text { (AMERICAN..., 2007) }\end{array}$ & $\begin{array}{c}60 \% \mathrm{C}_{3} \mathrm{~S} \\
14 \% \mathrm{C}_{2} \mathrm{~S}, 6 \% \mathrm{C}_{3} \mathrm{~A} \\
9 \% \mathrm{C}_{4} \mathrm{AF}\end{array}$ & 0.4 & 23 & 28 días & 0.057 & $\begin{array}{l}\text { Yodsudjai y } \\
\text { Wang } \\
(2013) \\
\end{array}$ \\
\hline $\begin{array}{l}\text { Cemento Portland } \\
\text { Tipo I ASTM C } 150 \\
\text { (AMERICAN..., 2007) }\end{array}$ & $\begin{array}{c}56.20 \% \mathrm{C}_{3} \mathrm{~S} \\
16.91 \% \mathrm{C}_{2} \mathrm{~S} \\
6.54 \% \mathrm{C}_{3} \mathrm{~A} \\
8.97 \% \mathrm{C}_{4} \mathrm{AF} \\
3.5 \% \text { gipso }\end{array}$ & 0.4 & 23 & 28 días & 0.058 & $\begin{array}{l}\text { Zhang et al. } \\
\text { (2013) }\end{array}$ \\
\hline $\begin{array}{l}\text { Cemento Portland } \\
\text { común }\end{array}$ & $\begin{array}{c}70 \% \mathrm{C}_{3} \mathrm{~S}, 9 \% \mathrm{C}_{2} \mathrm{~S} \\
3 \% \mathrm{C}_{3} \mathrm{~A}, \\
13 \% \mathrm{C}_{4} \mathrm{AF}, 3.3 \% \\
\text { gipso }\end{array}$ & 0.32 & 20 & $\begin{array}{c}140 \\
\text { horas }\end{array}$ & 0.048 & $\begin{array}{l}\text { Bouasker et } \\
\text { al. }(2014)\end{array}$ \\
\hline $\begin{array}{l}\text { Cemento Portland } \\
\text { común }\end{array}$ & $\begin{array}{l}59.08 \% \mathrm{C}_{3} \mathrm{~S}, \\
17.45 \% \mathrm{C}_{2} \mathrm{~S} \\
6.54 \% \mathrm{C}_{3} \mathrm{~A} \\
8.97 \% \mathrm{C}_{4} \mathrm{AF}\end{array}$ & 0.3 & 20 & 28 días & 0.050 & $\begin{array}{l}\text { Zhang et al. } \\
\text { (2013) }\end{array}$ \\
\hline $\begin{array}{l}\text { Cemento Portland } \\
\text { común }\end{array}$ & No indica & 0.4 & 20 & $\begin{array}{c}72 \\
\text { horas }\end{array}$ & 0.033 & $\begin{array}{l}\text { Liao y Wei } \\
(2014)\end{array}$ \\
\hline $\begin{array}{l}\text { Cemento Portland } \\
\text { Tipo I C } 150 \\
\text { (AMERICAN..., 2007) }\end{array}$ & No indica & 0.3 & 20 & $\begin{array}{c}72 \\
\text { horas }\end{array}$ & 0.037 & $\begin{array}{l}\text { Zuo y Wei } \\
(2015)\end{array}$ \\
\hline
\end{tabular}


La retracción química también puede ser expresada en forma de variación volumétrica haciendo uso de la Ecuación 2, que está en función del volumen de la retracción química, densidad del cemento y la relación a/c. Para ello, se divide la cantidad de retracción química entre el volumen de la mezcla. Así se elaboró la Figura 1, en la que se muestra una relación entre la retracción química expresada como variación volumétrica y la relación a/c para retracciones químicas de 0,$03 ; 0,05$ y $0,07 \mathrm{~mL} / \mathrm{g}$. Se observa que, para los todos los valores de retracción química evaluados, la variación volumétrica es siempre menor a $10 \%$. Asimismo, cuando la relación a/c aumenta, la variación volumétrica disminuye, siendo esta disminución más notoria para la mayor retracción química analizada. Por tanto, la magnitud de la retracción química expresada como variación volumétrica de la pasta de cemento depende de la relación a/c utilizada.

$$
\text { Ret Quim.vol. }=\frac{V_{\text {Ret. Quim. }} .}{V_{\text {Mezcla }}} \cdot 100=\frac{V_{\text {Ret. Quim. }}}{\frac{1}{\delta_{\text {cem }}}+\frac{a}{c}} \cdot 100
$$

Ec. 2

Donde:

Ret. Quim.vol es la retracción química expresada como variación volumétrica;

$V_{\text {Ret. Quím }}$ es el volumen de la retracción química;

$V_{\text {mezcla }}$ es el volumen de la mezcla entre el cemento y el agua;

$\delta_{c e m}$ es la densidad del cemento Portland; y

$a / c$ es la relación agua cemento.

Por otra parte, condiciones de curado - como temperatura y presión - afectan los valores de la retracción química. Con el aumento de la temperatura, aumenta la retracción química en edades iniciales (GEIKER; KNUDSEN, 1982; MOUNANGA et al., 2006; PANG et al., 2015) у cuando se aumenta la presión de curado, aumenta ligeramente la retracción química (PANG et al., 2015). Factores como composición mineralógica del cemento, distribución granulométrica y uso de materiales cementicios suplementarios también deben ser considerados. Más precisamente, la incorporación de adiciones minerales al cemento Portland modifica la cinética y las reacciones de hidratación $\mathrm{y}$, consecuentemente, la retracción química. Esta alteración depende de las características y composición de la adición mineral usada.

Siendo resultado de la hidratación, también puede ser directamente relacionada al calor de hidratación. Como ejemplo, en la Figura 2 de Lura, Winnefeld y Klemm (2010), se muestra una relación lineal entre la retracción química y el calor acumulado. Para el mismo tipo de cemento, esta relación es ligeramente afectada por la relación a/c. Los símbolos llenos indican que se colocó agua en la parte superior de la muestra, mientras que los símbolos vacíos muestran que no fue añadida agua. Para una relación a/c de 0.5, la correlación es muy buena, independientemente de si fue o no adicionada agua. Ya para una relación a/c de 0.3 , la correlación también es buena en el caso de los símbolos llenos, pero en edades avanzadas existe una divergencia de resultados en el caso de los símbolos vacíos.

La Figura 3 permite realizar una comparación entre las curvas de tasa de retracción química y flujo de calor con respecto al tiempo. Se observa similitud en el perfil de curva. Los períodos típicos de la cinética de hidratación son identificables en los dos resultados: período I, de pre-inducción; período II, de inducción; período III, de aceleración; y período IV, de desaceleración.

Figura 1 - Influencia da relación a/c en la retracción química, expresada como variación volumétrica $(\mathrm{v} / \mathrm{v}, \%)$, para retracciones químicas de $0,03,0.05$ y 0,07 en $\mathrm{mL} / \mathrm{g}$

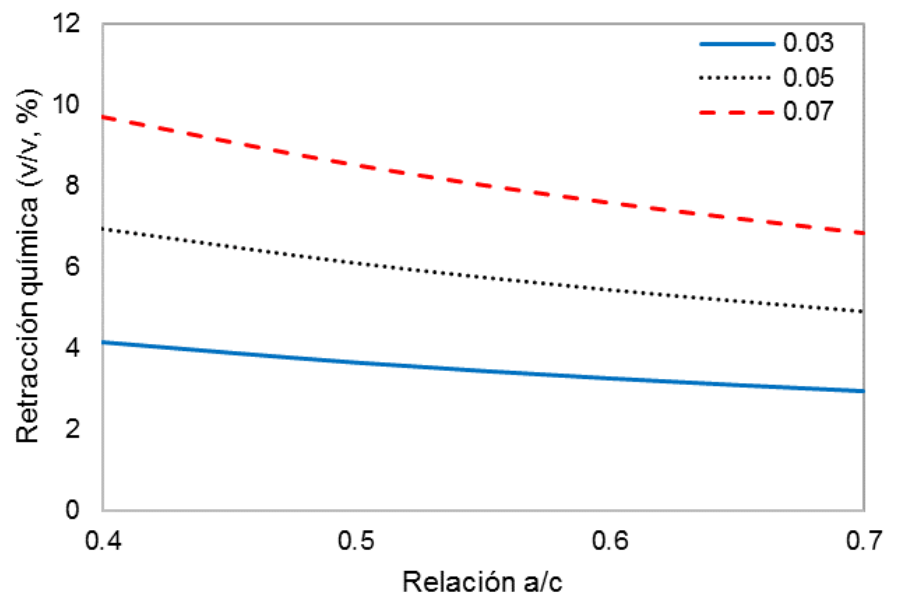


Figura 2 - Relación lineal entre la retracción química y el calor de hidratación

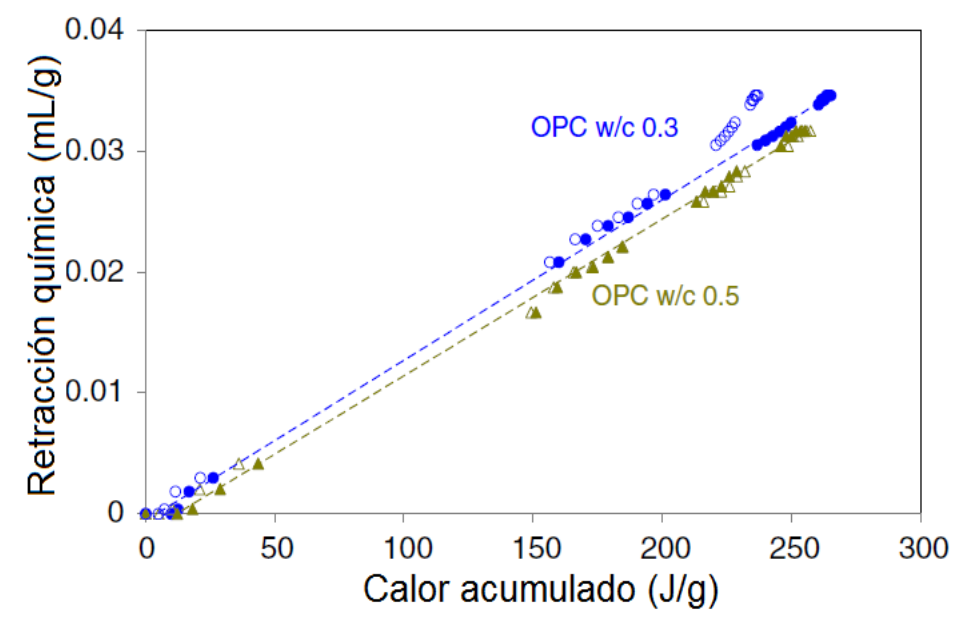

Fonte: Lura, Winnefeld y Klemm (2010).

Figura3 - (a) Curva de la tasa de retracción química y (b) Curva de flujo de calor

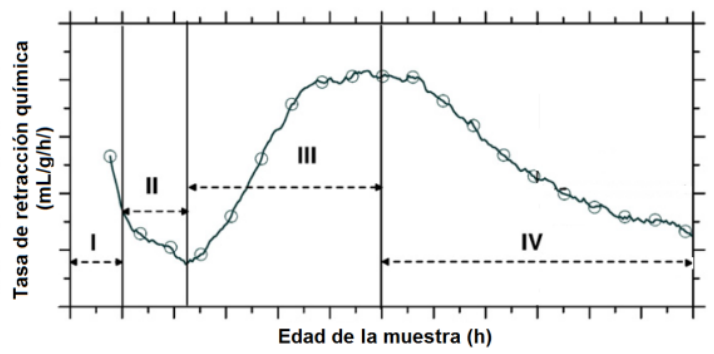

(a)

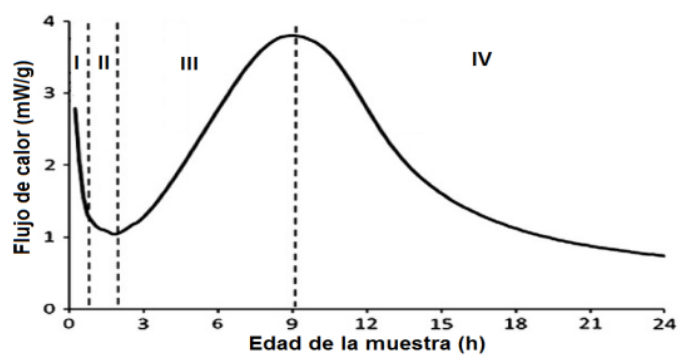

(b)

Fuentes: (a) Mindess, Young y Darwin (2003) y (b) Bullard et al. (2011).

\section{Métodos de determinación de la retracción química}

Como ya fue citado, la retracción química es determinada cuantificando la reducción de volumen que ocurre debido a las reacciones químicas de hidratación. Todos los métodos tienen en común que la muestra se mantiene saturada en agua (PARROTT et al., 1990; JUSTNES et al., 2000). Existen básicamente tres formas para determinar la retracción química: la dilatometría, la gravimetría y la picnometría (JUSTNES et al., 2000). Estos métodos varían en la forma en que se cuantifica la variación de volumen.

La revisión bibliográfica de normas internacionales muestra que la norma americana C-1608 (AMERICAN..., 2012) hace referencia a la determinación de la retracción química. Esta norma se basa en los estudios desarrollados por Geiker (1983 ${ }^{1}$ apud LURA; WINNEFELD; KLEMM, 2010) y Knudsen y Geiker (1985) y especifica los

${ }^{1}$ GEIKER, M. Studies of portland cement hydration: measurements of chemical shrinkage and a systematic evaluation of hydration curves by means of the dispersion métodos de dilatometría y gravimetría a los que denomina procedimientos A y B, respectivamente. Sin embargo, estudios como los de Costoya (2008), Pang et al. (2013) y Zhang et al.(2013) mencionan que esta norma presenta problemas, principalmente debido a la superficie de contacto de la pasta de cemento, ya que tiene gran influencia en los resultados; por otra parte, su reproductibilidad también está en discusión. A continuación, se detallan los métodos de: dilatometría, medición simultánea dilatometría-calorimetría, gravimetría y picnometría.

\section{Método de dilatometría}

En la Figura 4 se muestra un esquema de este procedimiento. Consiste en registrar la disminución del volumen que ocurre en una pasta de cemento en hidratación, midiendo el descenso de la columna de agua en una pipeta graduada que es colocada y ajustada por encima del frasco que contiene la muestra, a través de una tapa de hule. Inicialmente

model. Cophaggem, 1983. Technical University of Denmark, Cophagem, 1983. 
este método era complicado de realizar debido a que la lectura se realizaba de forma manual (JUSTNES et al., 2000). Esta limitación se resolvió con el uso de sensores digitales. Por ejemplo, Costoya (2008) utilizó una cámara web para registrar la caída de la columna de agua; por otra parte, Zhang et al. (2010) usaron sensores de presión para medir esta disminución

La Ecuación 3, muestra cómo se calcula la retracción química usando el método de dilatometría. La primera medida debe realizarse después de 60 minutos del contacto de los sólidos con el agua, como indica la norma C-1608 (AMERICAN..., 2012). Esta primera hora es necesaria para que el sistema alcance un equilibrio $\mathrm{y}$, así, se pueda iniciar con las lecturas.

$R Q(t)=\frac{h(t)-h(60 \mathrm{~min})}{M \text { cemento }}$

Donde:

$R Q(t)$ es la retracción química en un tiempo $t$ (mL/g $\mathrm{g}_{\text {cemento }}$ );

$h(t)$ es el nivel de agua en la pipeta en un tiempo $t$ $(\mathrm{mL})$;

$h(60 \mathrm{~min})$ es el nivel de agua en la pipeta en 60 minutos (primera lectura); $\mathrm{y}$

$M_{\text {cemento }}$ es la masa del cemento (g).

En la publicación de Justnes et al. (2000), se describe con más detalle este procedimiento experimental. Primero se midió la masa de los frascos vacíos y, posteriormente, la de los frascos con pasta, a fin de determinar la cantidad de material utilizado. Después, a la pasta se adicionó cuidadosamente agua destilada a temperatura ambiente hasta llenar el frasco, evitando perturbaciones en las muestras. Seguidamente, se usaron tapas de hule para conectar las pipetas a los frascos, teniendo cuidado para no generar burbujas de aire. A continuación, se llenó la pipeta con agua destilada. Se usaron pipetas graduadas de 0,2, 0,5 o $1 \mathrm{~mL}$, dependiendo de la variación de volumen esperado. En otros estudios como los de Costoya (2008) y Lura, Winnefeld y Klemm (2010), se colocó parafina líquida coloreada encima de la columna de agua de la pipeta, con el fin de minimizar la evaporación y facilitar la lectura. En seguida los recipientes fueron colocados en un baño térmico a una temperatura de $20 \pm 1^{\circ} \mathrm{C}$. Se leyó la posición del menisco de las pipetas a cada hora. A las 48 horas de hidratación, se obtuvo como resultado una retracción química de $0,03 \mathrm{~mL} / \mathrm{g}$, usando una relación a/c de 0,3, temperatura de 20 ${ }^{\circ} \mathrm{C}$ y frascos tipo Erlenmeyer.

También Lura (2003) utilizó este método, con los siguientes parámetros de ensayo: relación a/c de 0,37 , temperatura de $20{ }^{\circ} \mathrm{C}$, frascos cilíndricos de $25 \mathrm{~mm}$ de diámetro y $60 \mathrm{~mm}$ de altura (área de contacto de la muestra de $400 \mathrm{~mm}^{2}$ ) y $5 \mathrm{~g}$ de pasta por frasco. Con estos parámetros obtuvo una retracción química de $0,037 \mathrm{~mL} / \mathrm{g}$ a las 48 horas y de $0,045 \mathrm{~mL} / \mathrm{g}$ a los 7 días de hidratación.

Finalmente, en el estudio de Estrada (2016) se usó esta metodología con los siguientes parámetros de ensayo: relación a/c de 0,5 , espesor de la muestra de $20 \mathrm{~mm}$, temperatura del baño térmico de $25^{\circ} \mathrm{C}$ y frasco de 23,7 mm de diámetro y $47 \mathrm{~mm}$ de altura (área de contacto de $440 \mathrm{~mm}^{2}$ ). Estrada comparó estos resultados con los de la calorimetría de conducción isotérmica, manteniendo los mismos parámetros de ensayo. En la Figura 5 se muestran los resultados obtenidos: la tasa de retracción química es similar al flujo de calor, pudiéndose distinguir claramente sus principales eventos.

Figura 4 - Esquema de medición de la retracción química usando el método de la dilatometría

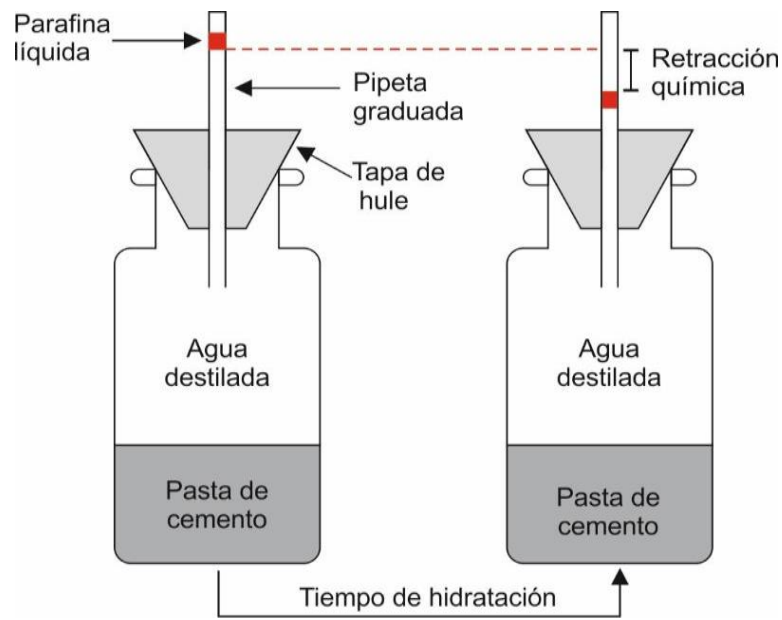


Figura 5 - Comparación de las curvas de tasa de retracción química con calorimetría de conducción isotérmica

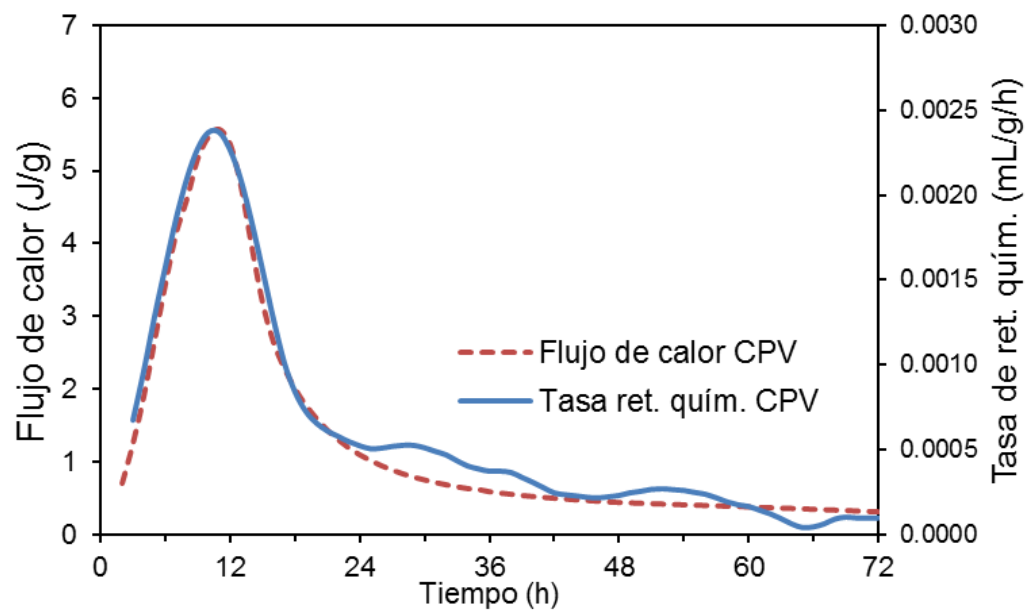

Fuente: Estrada (2016).

Las oscilaciones en los resultados a edades avanzadas pueden ser explicadas por efectos de dilatación del agua. Para minimizar el efecto de la temperatura en los resultados, es recomendable utilizar un baño térmico de precisión y realizar el ensayo en una sala con temperatura controlada.

\section{Método simultáneo, dilatometría- calorimetría}

Los ensayos de calorimetría de conducción isotérmica y de retracción química sirven para determinar la evolución de la cinética de hidratación en forma continua. Efectuarlos separadamente implica que los ensayos no se realizan bajo los mismos parámetros de medición, lo que sería totalmente garantizado al efectuar los dos ensayos simultáneamente y en el mismo equipo.

La determinación de la retracción química por medio del método de dilatometría y el ensayo de calorimetría de conducción isotérmica fue audazmente combinado en el estudio de Lura, Winnefeld y Klemm (2010), quienes efectuaron simultáneamente los dos ensayos con el objetivo de estudiar, al mismo tiempo, la evolución de la retracción química y del calor liberado durante el desarrollo de la hidratación. La Figura 6 muestra una fotografía del calorímetro usado con las pipetas insertas para medir la retracción química.

La principal diferencia de este procedimiento en comparación al ensayo de calorimetría, es que la superficie de la pasta fue cubierta con una película de agua desmineralizada y desaireada, de aproximadamente $0,5 \mathrm{~g}$, y que la superficie de contacto de la muestra fue de $400 \mathrm{~mm}^{2}$. Luego, se llenó el recipiente con aceite de parafina y se lo cerró con una tapa de hule, en la que se colocó una pipeta graduada de $1 \mathrm{~mL}$. Por su parte, en los ensayos de calorimetría no se adiciona nada sobre la pasta, sino que tan solo se tapa el frasco que contiene la pasta y se lo coloca en la celda del calorímetro.

En la Figura 2 de Lura, Winnefeld y Klemm (2010), se muestra una comparación de resultados de retracción química, para experiencias realizadas aisladamente y dentro del calorímetro. En este caso, se usaron los siguientes parámetros de ensayo: $6 \mathrm{~g}$ de pasta de cemento Portland común colocado en los mismos recipientes de vidrio utilizados en el ensayo de calorimetría. Esto resultó en una altura de 6 a $7 \mathrm{~mm}$ de pasta, además utilizaron relaciones a/c de 0,3 y 0,5 , temperatura constante de $20{ }^{\circ} \mathrm{C}$. Los resultados obtenidos para una edad de hidratación de 72 horas son: para el caso de las pastas elaboradas con una relación $\mathrm{a} / \mathrm{c}$ de 0,5 , una retracción química de $0,03 \mathrm{~mL} / \mathrm{g}$; mientras que, para las pastas elaboradas con a/c 0,3 la retracción fue de $0,035 \mathrm{~mL} / \mathrm{g}$.

Tal como fue descrito en el ítem correspondiente a retracción química, existe una proporcionalidad entre la retracción química y el calor acumulado, lo que demuestra que estos ensayos son comparables.

\section{Método de gravimetría}

Este método está basado en la medición indirecta de la variación de volumen y registra la disminución de flotabilidad del frasco que contiene la muestra y que está sumergido en un fluido, utilizando la ley de Arquímedes. Este procedimiento fue inicialmente 
propuesto por Rey $\left(1950^{2}\right.$ apud BOUASKER et al., 2008) y después fue adoptado y mejorado por Geiker y Knudsen (1982), Knudsen y Geiker (1985) y Paulini (1990). Esta técnica es más fácil de automatizar, pero también es más cara, debido a que se necesita una balanza de alta precisión para cada pasta a ser ensayada (LURA; WINNEFELD; KLEMM, 2010).

En la Ecuación 4 se describe la forma como se calcula la retracción química a partir de la variación de la masa (MOUNANGA et al., 2004; SANT; LURA; WEISS, 2006).

$$
V_{R Q}=\frac{\Delta V_{\text {pasta }}(t)}{g_{\text {cem }}}=\frac{M_{\text {sum. }}(t)-M_{\text {sum. }}(30)}{\rho_{\text {parafina }}} \cdot \frac{1}{g_{\text {cem }}}
$$

Dónde:

$\Delta V_{\text {pasta }}(\mathrm{mL})$ es la variación de volumen de la pasta;

$g_{c e m}(\mathrm{~g})$ es la cantidad de cemento en masa de la pasta;

$M_{\text {sum. }}(t)$ es la masa sumergida de la pasta en un tiempo t;

$M_{\text {sumergida }}(30)$ ( $\mathrm{g}$ ) es la masa sumergida 30 minutos después de la adición del agua (tiempo que es requerido debido al procedimiento experimental); $\mathrm{y}$

$\rho_{\text {parafina }}(\mathrm{g} / \mathrm{mL})$ es la densidad del aceite usado en el baño de flotabilidad, que aproximadamente es de $850 \mathrm{~kg} / \mathrm{m}^{3}$ a $23{ }^{\circ} \mathrm{C}$.

La publicación de Sant, Lura y Weiss (2006) describe con más detalle este procedimiento experimental. Primero se coloca la pasta de cemento recién mezclada en un recipiente de vidrio de $70 \mathrm{~mm}$ de diámetro y $50 \mathrm{~mm}$ de altura, como se muestra en la Figura 7; la masa típica usada en este ensayo es 25 g. Después, se vibran las muestras hasta lograr una cantidad de pasta uniformemente distribuida en la parte inferior del recipiente. Una vez completa la etapa de vibración, se coloca una capa de agua destilada en la superficie de la muestra con un gotero hasta alcanzar una capa uniforme de agua; en condiciones normales de ensayo se usan 10 $\mathrm{g}$ de agua. La cantidad de agua adicionada es importante por dos razones: la primera, porque abastecerá de líquido suficiente para compensar la variación de volumen que se espera; en segundo lugar, porque garantiza que toda la superficie de la pasta sea cubierta con agua durante el ensayo. Encima del agua se coloca aceite de parafina, primeramente, gota a gota, hasta que toda la superficie del agua sea cubierta y, después, hasta que el frasco quede casi lleno.

Figura 6 - Medición simultánea de calorimetría y de retracción química - a la izquierda, el recipiente usado con la pipeta inserta; a la derecha, el ensayo en conjunto

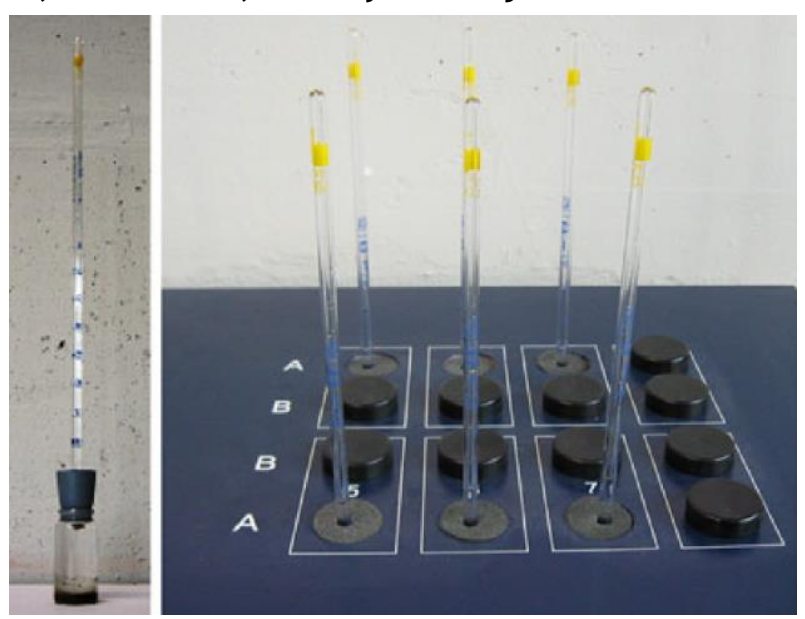

Fuente: Lura, Winnefeld y Klemm (2010).

${ }^{2}$ REY, M. Nouvelle Méthode de Mesure de L'hydratation des Liants Hydrauliques. Publication Techniques du CERILH, n. 31, 1950. 
Figura 7 - Procedimiento experimental en la medición de la retracción química

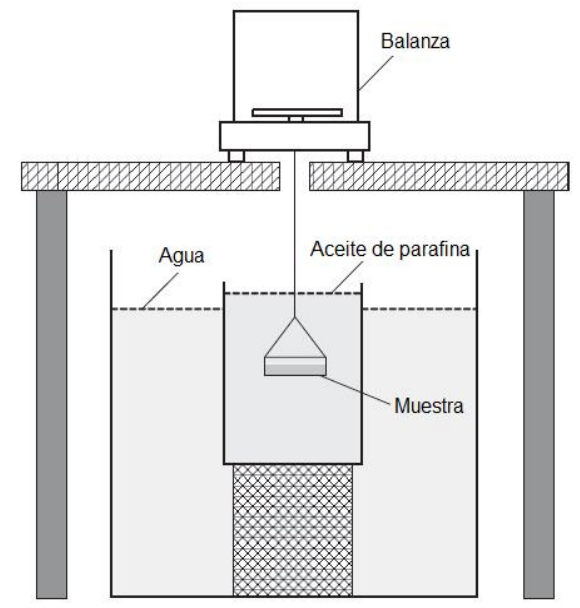

(a) Ilustración del método de flotabilidad Fuente: Sant, Lura y Weiss (2006).

En la parte inferior del recipiente se coloca una malla de acero inoxidable que se suspende mediante cuerdas de nylon con un gancho adaptado en la base de la balanza (Figura 7b). Luego se coloca cuidadosamente el frasco en un recipiente de plástico lleno de aceite de parafina de aproximadamente 5 litros. Para mantener constante la temperatura de la muestra, el recipiente de aceite de parafina fue sumergido en un baño térmico con temperatura regulada a $23 \pm 0,2{ }^{\circ} \mathrm{C}$ y con circulación de 25 litros de agua destilada. La mayor parte de la superficie del baño fue cubierta con una tapa de plástico para reducir la evaporación.

Se debe usar una balanza con precisión de $0,1 \mathrm{mg}$ o mayor y la variación en los resultados está influenciada por factores como: vibraciones en el baño térmico o la densidad del aceite de parafina. La variación de masa se registró automáticamente cada 5 minutos a partir del momento en que la muestra fue colocada en el baño térmico, aproximadamente 30 minutos después de la adicionar agua al cemento.

Usando esta metodología, Sant, Lura y Weiss (2006) obtuvieron una retracción química de 0,03 $\mathrm{mL} / \mathrm{g}$ para 48 horas de hidratación para los siguientes parámetros de ensayo: relación a/c de 0,3 , temperatura de $23{ }^{\circ} \mathrm{C}$, recipiente de $70 \mathrm{~mm}$ de diámetro y $50 \mathrm{~mm}$ de altura y $50 \mathrm{~g}$ de pasta.

\section{Método de picnometría}

Este método es solamente aplicable para determinar la retracción química total. Tiene similitud con el método de dilatometría y se lleva a cabo colocando una muestra de pasta de cemento dentro de un picnómetro y luego adicionando agua encima de la muestra. Para que el volumen del sistema se

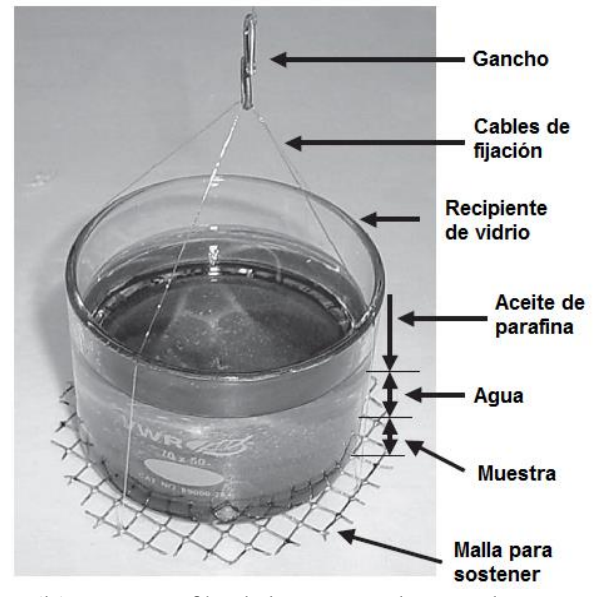

(b) Fotografía del cuerpo de prueba

mantenga constante, se vierte agua a intervalos regulares. El incremento de masa del sistema en conjunto es relacionado con la retracción química (JUSTNES et al., 2000; COSTOYA, 2008; GEIKER, 2017).

\section{Parámetros que influencian en la determinación de la retracción química}

Entre los parámetros que pueden influir en los resultados de la retracción química, tenemos: el espesor de la muestra, la relación a/c, el área superficial de la muestra, la cantidad de agua usada sobre la pasta y la composición del cemento utilizado.

\section{Espesor de la muestra}

Tazawa, Miyazawa y Kasai (1995) compararon espesores de pasta de 3, 6 y $12 \mathrm{~mm}$, usando una misma relación a/c de 0,3 y una temperatura de 20 ${ }^{\circ} \mathrm{C}$. Los autores observaron que a las 24 horas la retracción química es la misma sin importar el espesor de la pasta. Sin embargo, para 28 días, cuando se aumenta el espesor de la pasta, la retracción química medida disminuye.

Sant, Lura y Weiss (2006) realizaron estudios de medición de la retracción química de pastas de cemento Portland común con relación a/c de 0,3 ; frascos de vidrio de $70 \mathrm{~mm}$ de diámetro y $50 \mathrm{~mm}$ de altura; y espesores variados de la muestra en masa: 25,50 y 200 g (3, 6 y $24 \mathrm{~mm}$ de espesor, respectivamente). En la Figura 8 se muestran las curvas obtenidas, observándose que, para edades de hidratación mayor a 15 horas, la retracción química disminuye cuando se aumenta el espesor de la 
muestra. Asimismo, antes de este tiempo, el espesor de la muestra no tiene influencia significativa sobre la retracción química.

Por otra parte, Costoya (2008) estudió varias cantidades de pastas de alita, usando dos tipos de frascos: cilíndricos (de $20 \mathrm{~mm}$ de diámetro y $60 \mathrm{~mm}$ de altura) y Erlenmeyer (no especifica medidas). Las curvas que obtuvo se muestran en la Figura 9, donde se observa que existen dos familias de curvas: las tres superiores, pertenecen a los frascos cilíndricos y las tres inferiores a los frascos Erlenmeyer. Además, a partir de 10 horas, y cuando se aumenta la cantidad de muestra, la retracción química disminuye. Sin embargo, para edades de hidratación más cortos, la altura de la muestra no tiene influencia sobre las curvas de retracción.
Zhang et al. (2013) estudiaron el efecto del espesor de la pasta con cemento Portland común con los siguientes parámetros de ensayo: relación a/c de 0,4 y frasco de vidrio de $100 \mathrm{~mm}$ de diámetro y $150 \mathrm{~mm}$ de altura. Analizaron muestras de 6 diferentes espesores, desde 2,5 a $15 \mathrm{~mm}$ con incrementos sucesivos de $2,5 \mathrm{~mm}$, y concluyeron que, para edades menores a 24 horas, no se observaron diferencias significativas en la retracción química. Después de este tiempo, la retracción química de las pastas de bajo espesor incrementó rápidamente de $0,025 \mathrm{~mL} / \mathrm{g}$ para la edad de 24 horas, a $0,07 \mathrm{~mL} / \mathrm{g}$ para la edad de 28 días. Ya para espesores elevados el incremento fue lento - de $0,025 \mathrm{~mL} / \mathrm{g}$ para 24 horas de edad, a $0,05 \mathrm{~mL} / \mathrm{g}$ para 28 días de edad. Por su parte, Chen et al. (2013) verificaron el mismo comportamiento.

Figura 8 - Influencia de la altura de la muestra en la retracción química

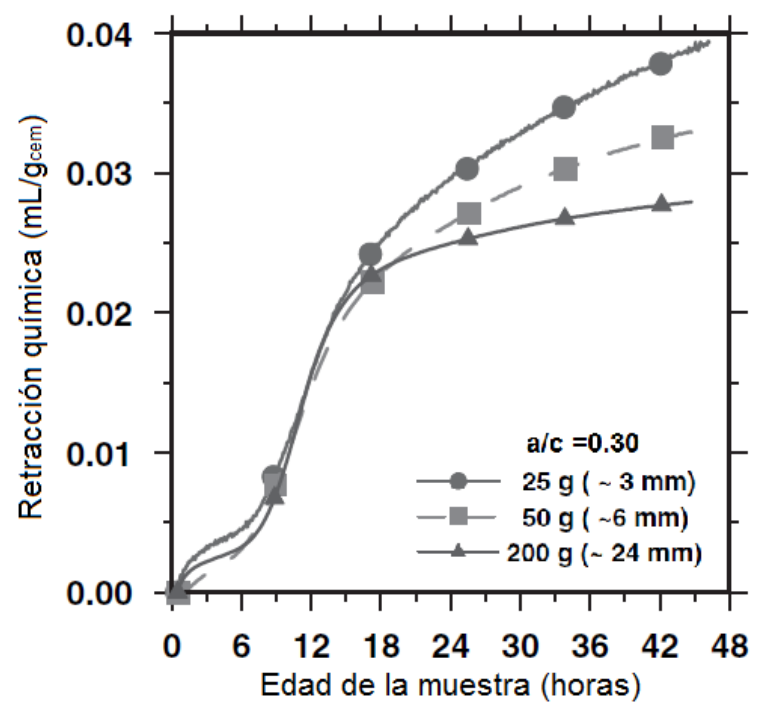

Fuente: Sant, Lura y Weiss (2006).

Figura 9 - Comparación de la retracción química para pastas de alita usando diferentes volúmenes medidos en frascos cilíndricos (c) y en Erlenmeyer (e)

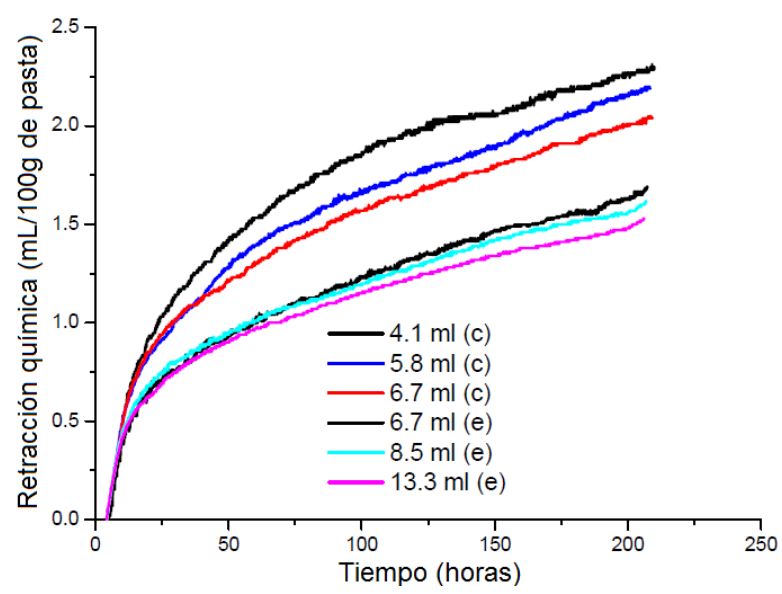

Fuente: Costoya (2008). 
De los párrafos anteriores se puede mencionar que, el aumento del espesor de la pasta de cemento causa una disminución en el valor de la retracción química, en edades de hidratación mayores a 10 o 15 horas. Este efecto puede ser explicado mediante dos hipótesis. La primera, al aumentar el espesor de la pasta se reduce la permeabilidad del sistema, consecuentemente, será impedido el ingreso de agua en el sistema para llenar los poros internos. La otra hipótesis es que, en pastas de poco espesor, un gran porcentaje se disuelve por el agua superficial, resultando en una tasa de hidratación rápida en edades avanzadas (SANT; LURA; WEISS, 2006; COSTOYA, 2008; PANG et al., 2015). El agua debe estar disponible para todo el espesor del sistema y la hidratación se debe realizar sin obstáculos, a fin de evitar el autosecado y así obtener una medida más exacta de la retracción química. Esto que se consigue ajustando el espesor de la pasta de cemento. La norma C-1608 (AMERICAN..., 2012) recomienda usar espesores entre 5 a $10 \mathrm{~mm}$, para ambos métodos.

Con el objetivo de mejorar la reproductibilidad y la precisión en los resultados del procedimiento $\mathrm{B}$ de la norma C-1608 (AMERICANA..., 2012) y Zhang et al. (2013) proponen insertar una barra magnética de agitación dentro del frasco con la intención de disminuir la influencia del espesor de la muestra. Esta barra actúa disminuyendo la formación de la estructura resistente de la pasta durante su hidratación. Utilizando esta técnica, los autores percibieron que el aumento del espesor de la pasta no tiene ninguna influencia sobre los resultados. Además, las velocidades de rotación entre $60 \mathrm{rpm}$ y 240 rpm no influyen en los resultados; sin embargo, los ensayos con rotación - por acelerar la hidratación - presentan mayor retracción química en comparación a los ensayos tradicionales (sin rotación) para edades mayores a 12 horas.

\section{Relación agua/cemento}

La norma C-1608 (AMERICAN..., 2012) recomienda usar una relación a/c próxima a 0,4 debido a que relaciones $\mathrm{a} / \mathrm{c}$ elevadas producen mayor exudación en las pastas de cemento, lo cual modifica la relación a/c efectiva (inicial) y, en consecuencia, influye en los resultados de retracción química (ZHANG et al., 2013). Por otra parte, al usar relaciones a/c bajas, las pastas presentan dificultades en su preparación, perjudicando la formación de una pasta adecuadamente homogénea. Además, la conectividad de la porosidad capilar tiende a ser discontinua, disminuyendo el transporte de iones (SANT; BENTZ; WEISS, 2011).

La Figura 10 muestra una correlación entre la relación a/c y el espesor de la muestra. Se observa que existe una región recomendada en la cual los valores de relación a/c y el espesor de la muestra no inciden sobre los resultados de la retracción química. Por lo tanto, ambos parámetros deben ser tomados en cuenta al momento de realizar el procedimiento experimental. Zhang et al. (2013) reportan que, al usar relaciones a/c bajas y espesores elevados, los resultados de retracción química presentan diferencias significativas; esto se debe a que en edades avanzadas el agua externa no puede permear fácilmente y llenar los poros capilares internos ya que la estructura de la pasta es muy densa.

\section{Figura 10 - Correlación entre la relación agua/cemento y el espesor de la muestra}

Fuente: Geiker (2017).

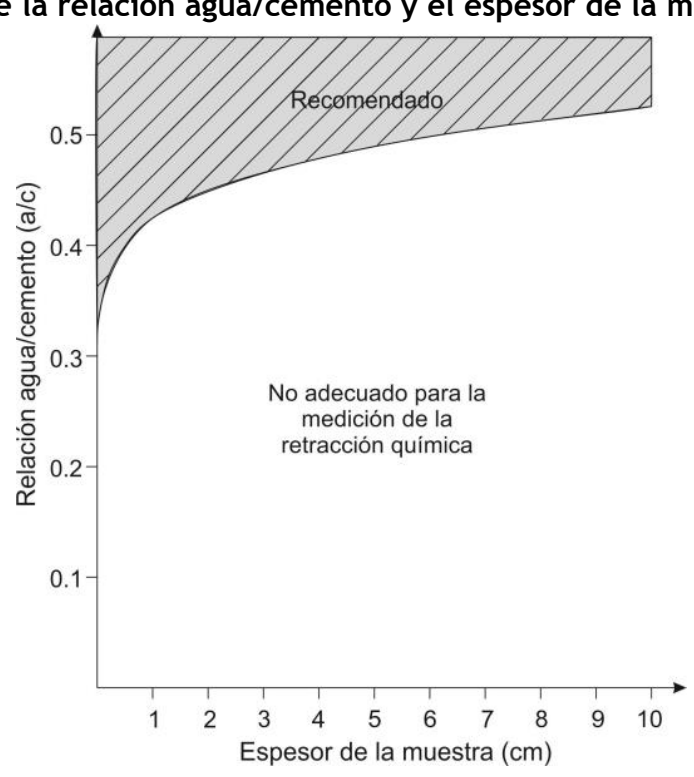




\section{Área superficial de la muestra}

El área superficial de la muestra también tiene influencia en los resultados de la retracción química. En la Figura 11 se muestran las curvas de retracción química que obtuvo Costoya (2008) y que evidencian la influencia que tiene el tamaño del área superficial de la muestra que está en contacto con el agua. Para verificar tal influencia en los resultados de la retracción química, el autor utilizó un recipiente cilíndrico de $2 \mathrm{~cm}$ de diámetro (menor área de contacto) y un recipiente tipo Erlenmeyer del cual no indicó el diámetro (mayor área de contacto). A continuación, colocó $6,7 \mathrm{~mL}$ de pasta de alita con una relación a/c de 0,4 en ambos tipos de frascos. Esto resultó en un espesor mayor de pasta para los recipientes cilíndricos y menor para los de tipo Erlenmeyer.

Los resultados muestran que en edades avanzadas mayores a 24 horas, y cuando la pasta tiene mayor área de contacto, los resultados de retracción química tienden a disminuir. Esto se debe a que al aumentar el diámetro de la pasta, el área de contacto crece, aumentando también la cantidad de productos hidratados en la superficie. Costoya (2008) encontró gran cantidad de portlandita [hidróxido de calcio, $\mathrm{Ca}(\mathrm{OH})_{2}$ ] precipitada en la superficie de la muestra que estuvo en contacto con el agua, lo cual modifica los resultados de retracción química. Es por ello que al realizar ensayos de retracción química se recomienda usar frascos con menor área de contacto.

\section{Cantidad de agua encima de la muestra}

Sant, Lura y Weiss (2006) estudiaron la influencia que tiene la cantidad de agua que se coloca por encima de las muestras en la retracción química. Observaron que, al incrementar la cantidad de agua sobre la superficie de la pasta de cemento de 10 a $40 \mathrm{~g}, \quad$ los resultados de retracción química aumentan. Esto se debe a que, cuando se tiene mayor cantidad de agua, la muestra se disuelve más rápido, y se aceleran las reacciones de hidratación y también la retracción química.

Para evitar que la cantidad de agua sobre la muestra influya en los resultados de retracción química, se sugiere:

(a) para el método de dilatometría - tal como en los estudios de Lura, Winnefeld y Klemm (2010) o Estrada (2016) - usar una cantidad mínima de agua sobre la muestra - alrededor de 1 o $2 \mathrm{~g}$; lo necesario para garantizar la correcta saturación de las pastas durante el ensayo y rellenar el resto del frasco con aceite de parafina. Cabe mencionar que en estudios como los de Costoya (2008), Kocaba (2009) y Berodier (2015) se adiciona agua hasta llenar la pipeta y solamente se coloca una pequeña cantidad de aceite de parafina en la parte superior de la pipeta para evitar evaporación; y

(b) para el método de gravimetría, tal como sugiere Geiker (2017), la cantidad de agua debe ser limitada y debe corresponder al valor máximo de retracción química calculado; además se debe colocar algunas gotas de agua para humedecer la superficie del frasco.

Figura 11 - Comparación de curvas de retracción química obtenidas usando recipientes cilíndricos y del tipo Erlenmeyer

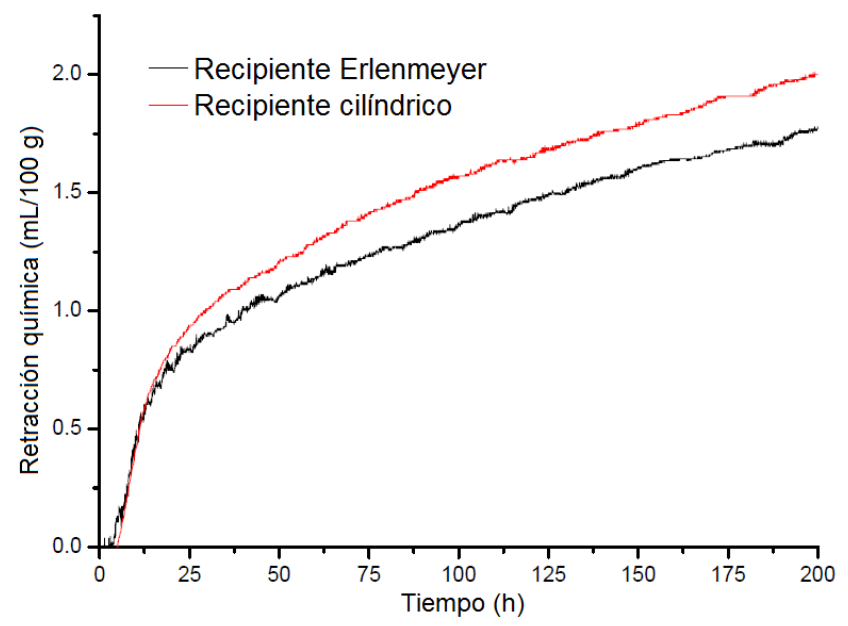

Fuente: Costoya (2008) 
Por otro lado, la norma C-1608 (AMERICAN..., 2012) indica que al colocar agua destilada sobre la pasta de cemento, el vertido se debe realizar de forma cuidadosa y sin causar disturbios en la superficie de la pasta. Sin embargo, en la práctica, al adicionar agua es inevitable causar tales disturbios en la superficie de la pasta. Por esto se recomienda adicionar cuidadosamente el agua gota a gota- para minimizar disturbios en la superficie de la pasta.

\section{Composición del cemento}

Yodsudjai y Wang (2013) estudiaron cómo es afectada la retracción química usando cementos con diferentes composiciones. Para ello, emplearon una relación a/c constante de 0,4 durante 28 días. Encontraron que la composición de los cementos influye en la retracción química de las pastas cementicias. Cuando se aumenta el contenido de la fase aluminatos - es decir, aluminato tricálcico $\left(\mathrm{C}_{3} \mathrm{~A}\right)$ y ferroaluminatotetracálcico $\left(\mathrm{C}_{4} \mathrm{AF}\right)$ - la retracción química también aumenta. Así mismo, el incremento del equivalente alcalino $\left(\% \mathrm{Na}_{2} \mathrm{O}+\right.$ $0.658 \% \quad \mathrm{~K}_{2} \mathrm{O}$ ) hace que aumente la retracción química debido a que la presencia de álcalis acelera la hidratación del cemento.

Holt (2005) y Bentz, Sant y Weiss (2008) reportan que la finura del cemento es un factor importante que tiene influencia en los resultados de la retracción química debido a su mayor área de contacto con el agua de mezclado. Bouasker (2008) menciona que el fíler calcáreo presente en el cemento Portland acelera la retracción química, sobre todo en edades menores a 24 horas; sin embargo, la magnitud de la retracción química no se altera significativamente. Las adiciones minerales presentes en los cementos o agregadas separadamente producen un incremento en el desarrollo de la retracción química (KOCABA; GALLUCCI; SCRIVENER, 2012; BERODIER, 2015; SCHÖLER et al., 2015; SCRIVENER; JUILLAND; MONTEIRO, 2015).

\section{Conclusiones}

Cuantificar la retracción química es útil para determinar la cinética y el grado de hidratación de materiales cementicios. El calor de hidratación y la retracción química son consecuencia directa de la reacciones de hidratación; ambos son ensayos de acompañamiento de la hidratación y pueden relacionarse entre sí. Debido a que las reacciones de hidratación son más significativas en edades iniciales, la tasa de retracción química presenta la misma forma de curva que el flujo de calor liberado.
En este trabajo se presentaron las técnicas para medir la retracción química: dilatometría, dilatometría-calorimetría, gravimetría y picnometría. Los parámetros que influyen en los resultados y que deben ser considerados son: espesor de pasta, relación a/c, área superficial de la muestra, cantidad de agua sobre la muestra, composición del cemento utilizado.

La ventaja de usar el método de dilatometría es que utiliza equipos de bajo costo y es posible registrar la retracción química de varias muestras a la vez, aprovechando las celdas del calorímetro (al igual que en el método de dilatometría-calorimetría). Por otro lado, el método gravimétrico requiere una balanza de alta precisión por cada muestra evaluada; además tiene alta inestabilidad debido a que el frasco que contiene la muestra está suspendido en un medio líquido y propenso a vibraciones.

Las condiciones óptimas de ensayo son: para el método de dilatometría: usar espesores de muestra pequeños y cercanos a $10 \mathrm{~mm}$, la forma del frasco debe ser cilíndrico con área de contacto cercano a $400 \mathrm{~mm}^{2}$; para el método gravimétrico: espesor de la muestra de $3 \mathrm{~mm}$ y área de contacto de $3800 \mathrm{~mm}^{2}$. Para ambos métodos la relación a/c debe ser cercana a 0,4 y también es importante usar un baño térmico de precisión y sala de temperatura controlada.

Los ensayos de retracción química permiten estudiar la cinética de hidratación de cementos sin que sea necesario adquirir dispendiosos calorímetros isotérmicos.

\section{Referencias}

AMERICAN SOCIETY FOR TESTING AND MATERIALS. C-150: standard specification for Portland cement. West Conshohocken, 2007.

AMERICAN SOCIETY FOR TESTING AND MATERIALS. C-1608: standard test method for chemical shrinkage of hydraulic cement paste. West Conshohocken, 2012.

BENTZ, D. P. Three-Dimensional Computer Simulation of Portland Cement Hydration and Microstructure Development. Journal of the American Ceramic Society, v. 80, n. 1, p. 3-21, 1 jan. 1997.

BENTZ, D. P.; LURA, P.; ROBERTS, J. W. Mixture Proportioning for Internal Curing. Concrete International, v. 27 , n. 2, p. $35-40$, 2005.

BENTZ, D. P.; SANT, G.; WEISS, J. Early-Age Properties of Cement-Based Materials. I: Influence of Cement Fineness. Journal of Materials in Civil Engineering, v. 20, n. 7, p. 502-508, 2008. 
BERODIER, E. Impact of the Suplementary Cementitious Materials on the Kinetics and Micoestruture Development of Cement Hydration. 2015. École Polytechnique Fédérale de Lausanne, Suiza, 2015.

BOUASKER, M. et al. Early-Age Deformation and Autogenous Cracking Risk of Slag-Limestone Filler-Cement Blended Binders. Construction and Building Materials, v. 55, p. 158-167, 31 mar. 2014.

BOUASKER, M. et al. Chemical Shrinkage of Cement Pastes and Mortars at Very Early Age: Effect of limestone filler and granular inclusions. Cement and Concrete Composites, v. 30, n. 1, p. 13-22, jan. 2008.

BULLARD, J. W. et al. Mechanisms of Cement Hydration. Cement and Concrete Research, v. 41, n. 12, p. 1208-1223, dez. 2011.

CHEN, H. et al. Prediction of Self-Desiccation in Low Water-to-Cement Ratio Pastes Based on Pore Structure Evolution. Cement and Concrete Research, v. 49, p. 38-47, jul. 2013.

COSTOYA, F. M. M. Effect of Particle Size on the Hydration Kinetics and Microstructural Development of Tricalcim Silicate. 2008. École Polytechnique Fédérale de Lausanne, Suiza, 2008.

ESTRADA, D. P. Influencia del Metacaolin en las Propiedades en Estado Fresco del Cemento Portland: hidratación y retracción química. São Paulo, 2016. Tese (Dissertação em Engenharia Civil) - Escola de Engenharia, Universidade de São Paulo, São Paulo, 2016.

GEIKER, M. Characterisation of Development of Cement Hydration Using Chemical Shrinkage. In: SCRIVENER, K.; SNELLINGS, R.; LOTHENBACH, B. A Practical Guide to Microstructural Analisis of Cementitious Materials. Boca Raton: CRC Press, 2017.

GEIKER, M.; KNUDSEN, T. Chemical Shrinkage of Portland Cement Pastes. Cement and Concrete Research, v. 12, n. 5, p. 603-610, set. 1982.

HOLT, E. Contribution of Mixture Design to Chemical and Autogenous Shrinkage of Concrete at Early Ages. Cement and Concrete Research, v. 35, n. 3, p. 464-472, mar. 2005.

JENSEN, O. M.; HANSEN, P. F. Water-Entrained Cement-Based Materials: I. Principles and theoretical background. Cement and Concrete Research, v. 31, n. 4, p. 647-654, abr. 2001b.

JENSEN, O. M.; HANSEN, P. F. Autogenous Deformation and RH-Change in Perspective. Cement and Concrete Research, v. 31, n. 12, p. 1859-1865, dez. 2001a.
JUSTNES, H. et al. Chemical Shrinkage of Cement Pastes With Plasticizing Admixtures. Nordic Concrete Research, v. 54, p. 24-39, 2000.

KNUDSEN, T.; GEIKER, M. Obtaining Hydration Data by Measurement of Chemical Shrinkage With an Archimeter. Cement and Concrete Research, v. 15, n. 2, p. 381-382, mar. 1985.

KOCABA, V. Development and Evaluation of Methods to Follow Microstructural Development of Cementitious Systems Including Slags. 2009. École Polytechnique Fédérale de Lausanne, Suiza, 2009.

KOCABA, V.; GALLUCCI, E.; SCRIVENER, K. L. Methods for Determination of Degree of Reaction of Slag in Blended Cement Pastes. Cement and Concrete Research, v. 42, n. 3, p. 511-525, mar. 2012.

HEWLETT, P. LEA'S Chemistry of Cement and Concrete. $4^{\text {th }}$. ed. New York: Wiley, 1998.

LIAO, Y.; WEI, X. Relationship Between Chemical Shrinkage and Electrical Resistivity for Cement Pastes at Early Age. Journal of Materials in Civil Engineering, v. 26, n. 2, p. 384-387, 2014.

LURA, P. Autogenous Deformation and Internal Curing of Concrete. 2003. Delft DUP Science, Netherland, 2003.

LURA, P. et al. Early-Age Acoustic Emission Measurements in Hydrating Cement Paste: evidence for cavitation during solidification due to self-desiccation. Cement and Concrete Research v. 39, n. 10, p. 861-867, out. 2009.

LURA, P.; JENSEN, O. M.; VAN BREUGEL, K. Autogenous Shrinkage in High-Performance Cement Paste: an evaluation of basic mechanisms. Cement and Concrete Research, v. 33, n. 2, p. 223-232, 2003.

LURA, P.; WINNEFELD, F.; KLEMM, S. Simultaneous Measurements of Heat of Hydration and Chemical Shrinkage on Hardening Cement Pastes. Journal of Thermal Analysis and Calorimetry, v. 101, n. 3, p. 925-932, 2010.

MINDESS, S.; YOUNG, J. F.; DARWIN, D. Concrete. New Jersey: Prentice Hall, 2003.

MOUNANGA, P. et al. Autogenous Deformations of Cement Pastes: part I: temperature effects at early age and micro-macro correlations. Cement and Concrete Research, v. 36, n. 1, p. 110-122, jan. 2006. 
MOUNANGA, P. et al. Early-Age Autogenous Cracking of Cementitious Matrices: physicochemical analysis and micro/macro investigations. Materials and Structures/Materiaux et Constructions, v. 44, n. 4, p. 749-772, 2011.

MOUNANGA, P. et al. Predicting $\mathrm{Ca}(\mathrm{OH}) 2$ Content and Chemical Shrinkage of Hydrating Cement Pastes Using Analytical Approach. Cement and Concrete Research, v. 34, n. 2, p. 255-265, 2004.

NEVILLE, A. M.; BROOKS, J. J. Tecnologia do Concreto. 2. ed. Porto alegre: Bookman, 2007.

PANG, X. et al. A Comparison Study of Portland Cement Hydration Kinetics as Measured by Chemical Shrinkage and Isothermal Calorimetry. Cement and Concrete Composites, v. 39, p. 2332, maio 2013.

PANG, X. et al. An Innovative Test Apparatus for Oil Well Cement: in-situ measurement of chemical shrinkage and tensile strength. Construction and Building Materials, v. 74, p. 93-101, 2015.

PARROTT, L. J. et al. Monitoring Portland cement hydration: Comparison of methods. Cement and Concrete Research, v. 20, n. 6, p. 919-926, nov. 1990.

PAULINI, P. Reaction Mechanisms of Concrete Admixtures. Cement and Concrete Research, v. 20, n. 6, p. 910-918, 1990.

SANT, G.; BENTZ, D.; WEISS, J. Capillary Porosity Depercolation in Cement-Based Materials: measurement techniques and factors which influence their interpretation. Cement and Concrete Research, v. 41, n. 8, p. 854-864, ago. 2011.

SANT, G.; LURA, P.; WEISS, J. Measurement of Volume Change in Cementitious Materials at Early Ages. Review of Testing Protocols and Interpretation of Results. Transportation Research Board, v. 1979, p. 21-29, 2006.
SCHÖLER, A. et al. Hydration of Quaternary Portland Cement Blends Containing blAst-Furnace Slag, Siliceous Fly Ash and Limestone Powder. Cement and Concrete Composites, v. 55, p. 374382, jan. 2015.

SCRIVENER, K. L.; JUILLAND, P.;

MONTEIRO, P. J. M. Advances in Understanding Hydration of Portland Cement. Cement and Concrete Research, v. 78, Part A, p. 38-56, dez. 2015.

TAZAWA, E. Autogenous Shrinkage of Concrete. London: E FN Spon, 1999.

TAZAWA, E.; MIYAZAWA, S.; KASAI, T. Chemical Shrinkage and Autogenous Shrinkage of Hydrating Cement Paste. Cement and Concrete Research, v. 25, n. 2, p. 288-292, fev. 1995.

YODSUDJAI, W.; WANG, K. Chemical Shrinkage Behavior of Pastes Made With Different Types of Cements. Construction and Building Materials, v. 40, p. 854-862, mar. 2013.

ZHANG, J. et al. Early Hydration and Setting of Oil Well Cement. Cement and Concrete Research, v. 40, n. 7, p. 1023-1033, 2010.

ZHANG, T. et al. Measurement of Chemical Shrinkage of Cement Paste: comparison study of ASTM C 1608 and an improved method.

Construction and Building Materials, v. 48, p. 662-669, nov. 2013.

ZUO, Y.; WEI, X. Relations Among Electrical Resistivity, Chemical and Autogenous Shrinkage of Cement Pastes. Advances in Cement Research, v. 27, n. 3, p. 175-183, 2015.

\section{Agradecimientos}

Los autores agradecen a CAPES por el apoyo económico para la realización de este trabajo. 


\section{Delver Plinio Estrada Caceres}

Departamento de Engenharia de Construção Civil, Escola Politécnica | Universidade de São Paulo | Av Almeida Prado, Trav. 2, 87, Cidade Universitária | São Paulo - SP - Brasil | CEP 05508-900 | Tel.: (11) 3091-5459 | E-mail: delver.estrada@usp.br

\section{Vanderley Moacyr John}

Departamento de Engenharia de Construção Civil, Escola Politécnica | Universidade de São Paulo | Tel.: (11) 3091-5794 |

E-mail: vmjohn@lme.pcc.usp.br

\section{Maria Alba Cincotto}

Departamento de Engenharia de Construção Civil, Escola Politécnica | Universidade de São Paulo | Tel.: (11) 3091-5544 |

E-mail: cincotto@usp.br

\section{Revista Ambiente Construído}

Associação Nacional de Tecnologia do Ambiente Construído

Av. Osvaldo Aranha, $99-3^{\circ}$ andar, Centro

Porto Alegre - RS - Brasil

CEP $90035-190$

Telefone: +55 (51) 3308-4084

Fax: +55 (51) 3308-4054

www.seer.ufrgs.br/ambienteconstruido

E-mail: ambienteconstruido@ufrgs.br 